\title{
Parametric Fault Diagnosis of an Active Gas Bearing
}

Sekunda, André; Niemann, Henrik; Poulsen, Niels Kjølstad; Santos, IImar

Published in:

International Journal of Control, Automation and Systems

Link to article, DOI:

10.1007/s12555-017-0738-2

Publication date:

2019

Document Version

Peer reviewed version

Link back to DTU Orbit

Citation $(A P A)$ :

Sekunda, A., Niemann, H., Poulsen, N. K., \& Santos, I. (2019). Parametric Fault Diagnosis of an Active Gas Bearing. International Journal of Control, Automation and Systems, 17(1), 69-84. https://doi.org/10.1007/s12555017-0738-2

\section{General rights}

Copyright and moral rights for the publications made accessible in the public portal are retained by the authors and/or other copyright owners and it is a condition of accessing publications that users recognise and abide by the legal requirements associated with these rights.

- Users may download and print one copy of any publication from the public portal for the purpose of private study or research.

- You may not further distribute the material or use it for any profit-making activity or commercial gain

- You may freely distribute the URL identifying the publication in the public portal

If you believe that this document breaches copyright please contact us providing details, and we will remove access to the work immediately and investigate your claim 


\title{
Parametric Fault Diagnosis of an Active Gas Bearing
}

\author{
André Sekunda*, Henrik Niemann, Niels Kjølstad Poulsen and Ilmar Santos
}

\begin{abstract}
Recently research into active gas bearings has had an increase in popularity. There are several factors that can make the use of gas bearings favourable. Firstly gas bearings have extremely low friction due to the usage of gas as the lubricant which reduce the needed maintenance. Secondly gas bearings is a clean technology which makes it possible to use for food processing, air condition and applications with similar requirements. Active gas bearings are therefore useful for applications where downtime is expensive and dirty lubricants such as oil are inapplicable. In order to keep as low downtime as possible it is important to be able to determine when a fault occurs. Fault diagnosis of active gas bearings is able to minimize the necessary downtime by making certain the system is only taken offline when a fault has occurred. Usually industry demands the removal of any sensor redundancy in systems. This makes it impossible to isolate faults using passive fault diagnosis. Active fault diagnosis methods have been shown able to isolate faults when there is no sensor redundancy. This makes active fault diagnosis methods relevant for industrial systems. It is in this paper shown possible to apply active fault diagnosis to diagnose parametric faults on a controllable gas bearing. The fault diagnosis is based on a statistical detector which is able to quantify the quality of the diagnosis scheme.
\end{abstract}

Keywords: Active Fault Diagnosis, Active gas bearing, Parametric Faults, Laboratory Experiment, Closed Loop Fault Diagnosis

\section{INTRODUCTION}

Control of active radial gas bearings by means of piezoelectric actuation is an interesting subject that can significantly extend gas bearing technology in the framework of industry 4.0. Until recently all multiphysical mathematical models derived based on the first principles, linking fundamental equations coming from the fields of rotordynamics, fluid dynamics, material science and control techniques, presented considerable deviations from experimental results, as it can be concluded from [1-5]. Not long ago there wasn't any proper models available for control design of active radial gas bearings via piezoelectric actuation. Pierart and Santos [6-9] developed accurate mathematical models based on the first principles leading to high order models consisting 144 states. After model reduction, it was shown by the authors to be possible to use them to effectively design model-based controllers. Lately it was shown by [10] that identification of low order models of the position of a shaft held in place by an active gas bearing using a 6âĂŹth order linear model is possible. With such a model it is possible to design advanced controllers such as done in [10]. The goal of all the controllers applied to the gas bearings has been to reject external disturbances. This is possible as long as the system is not changing. Fault detection is thus relevant to determine when and how the system changes such that appropriate action can be conducted and performance regained. It has previously been shown how to detect and isolate faults for several different mechanical systems such as induction engines [11], gearboxes [12] and bearings [13]. In all these cases the goal of the fault diagnosis was to regain performance or stop the system before it broke down.

Fault diagnosis can be divided into two subcategories, active and passive fault detection. Where passive fault diagnosis makes it possible to diagnose faults by information gained from redundancy, active fault detection introduces an excitation signal in order to gain information usable for diagnosis of faults. The gas bearing system used in this paper has a fixed non-redundant number of sensors which can become problematic for fault diagnosis based on passive fault diagnosis. For such a system, as the active gas bearing, isolation of several faults using passive fault diagnosis can become unrealistic to achieve. Active fault detection add information, through an excitation signal, to the diagnosis scheme and is therefore seen as a more appropriate approach for fault diagnosis of the active gas bearing.

Active fault diagnosis has been studied extensively with

André Sekunda and Henrik Niemann are with the Department of Electrical Engineering, Automation and Control, Tech. University of Denmark, Denmark (e-mails: \{aksek, hhn\}@elektro.dtu.dk). Niels Kjølstad Poulsen is with the Department of Applied Mathematics and Computer Science, Tech. University of Denmark, Denmark (e-mails: nkpo@dtu.dk). Ilmar Santos is with the Department of Mechanical Engineering, Tech. University of Denmark, Denmark (e-mails: ifs@mek.dtu.dk).

* Corresponding author. 
the research branching into two directions. One branch treats the fault diagnosis problem as a discrete bank of different possible systems and compares the measured output with that predicted given each of the systems in the bank. One of the systems represents the healthy system while the others represent the system subject to one of the possible faults. Extensive effort has been put into defining the framework and the optimal input for the diagnosis of such a problem statement [14-20]. Another approach for defining the effect of the fault has been proposed in [21]. Here a fault signature system is designed and the effect of each of the faults on the fault signature system is instead used for diagnosis [22,23]. The approach is based on the system being part of a closed loop scheme and automatically decouple the noise from the residual. The active gas bearing should always have an controller active when faults are introduced due to safety where this feature becomes extra convenient.

The main contribution of this paper is to diagnose faults on an active gas bearing. The active gas bearing test rig used is a multiple input multiple output system with two inputs(piezo actuators) and two measured outputs(displacement sensors). It is shown possible to diagnose faults occurring both on the piezo actuators and the displacement sensors using a non-redundant sensor setup. The fault detection is conducted by designing a fault signature system and using a matched filter detector for diagnosis of the faults. The method was proposed in [24] where examples using a simple second order system were given. There it was shown that the method makes it easy to determine the impact of the feedback loop for closed loop systems. All faults considered are here modelled as parametric faults. It is here shown possible to successfully diagnose different faults for the gas bearing under non-rotating as well as rotating conditions. Furthermore experiments have been conducted using both a simple proportional controller as well as a linear-quadratic controller. Lastly a whitening filter proposed in [24] was implemented and shown able to whiten the noise on the residual signal. The whitening of the noise makes the residual into a standard form for design of statistical detectors.

This paper is structured as follows: In Section 2. the model of the gas bearing is introduced together with the identification results; In Section 3. the controller design and augmented controller scheme is presented; In Section 4. the parametric faults considered are defined; In Section 5. an analysis of the excitation signal design is conducted; In Section 6. the detector design is presented; In Section 5. an analysis of the excitation signal design is conducted; In Section 7. the experimental results are presented, and finally, in Section 8., a discussion of the results and future possible improvements are given.

\section{MODEL STRUCTURE}

An active gas bearing is used for the experimental work conducted in this paper. A schematic of the gas bearing considered is shown on Figure 1.

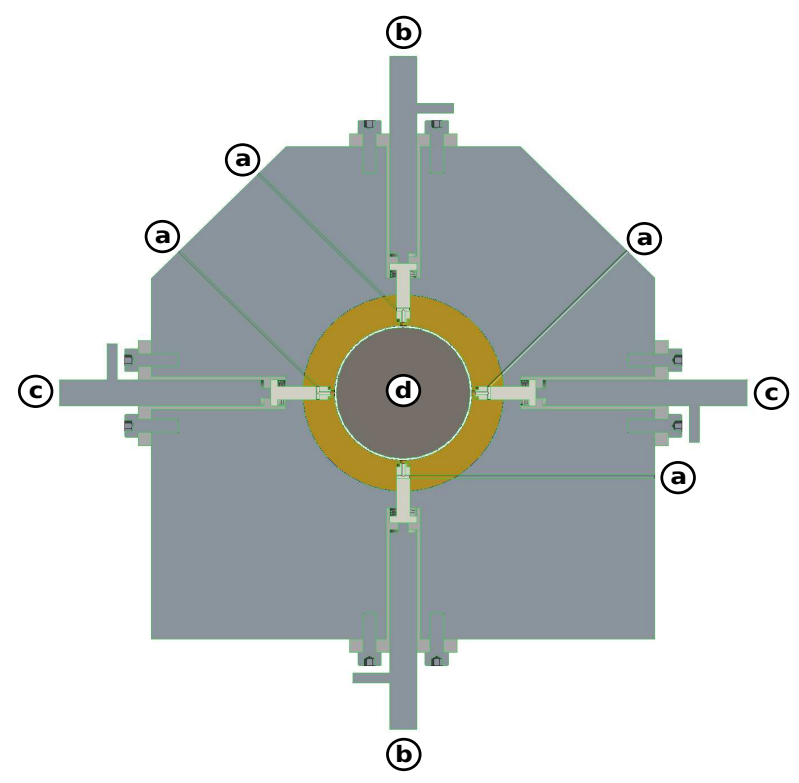

Fig. 1. A cross section schematic of the active gas bearing. The air is pumped in through the 4 inlets denoted by $\mathbf{a}$. The piezo actuators denoted $\mathbf{c}$ open and close the flow of air from the horizontal direction. Likewise the piezo actuators denoted $\mathbf{c}$ open and close the flow of air towards the shaft $\mathbf{d}$ in the vertical direction.

The vertical piezo actuators denoted $\mathbf{b}$ and the horizontal piezo actuators denoted $\mathbf{c}$ on Figure 1 have each respectively been lumped together as a single controllable input. The goal is to keep a rotor in place using only the active gas bearing. This is done by pumping air into the gas bearing through the tubes $\mathbf{a}$ using the piezo actuators $\mathbf{b}$ and $\mathbf{c}$. The rotor and gas bearing are connected by a flexible shaft. The horizontal and vertical positions of the rotor are measured and used for feedback control. A picture of the experimental test rig is shown on Figure 2 where the location of the displacement sensors and actuators can be seen.

A low order physical model structure was identified in [25] which can describe the main dynamics. The model describe the dynamics from the piezo actuators to the position of the disc. It was shown that the model depends on the rotational speed of the rotor and injection pressure of the lubricant injected into the bearing gap. In this work a constant rotational speed and fixed pressure of the gas in 
the active gas bearing is chosen for each experiment. The state vector is defined in equation (1).

$$
x_{6 x 1}=\left[\begin{array}{c}
l \\
i \\
\mathcal{M}
\end{array}\right]
$$

Here $l$ is a column vector denoting the lateral displacement of the rotor in the horizontal and vertical direction respectively. $\mathcal{M}$ is a column vector denoting the state of the actuator dynamics described by the linear position of the piezo electric stacks. Let the system be described by the standard state space description given by equation (2).

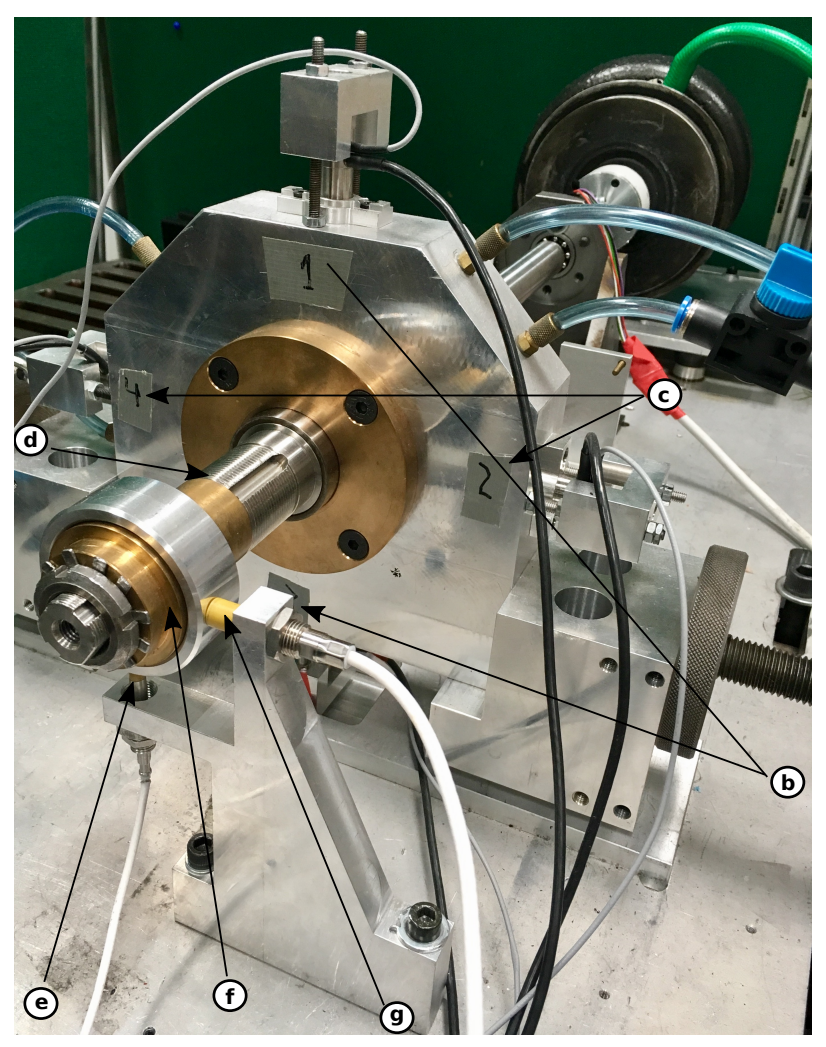

Fig. 2. Picture of the experimental test rig used for conducting active fault detection. The different parts of the test rig are as follows: b are the piezo actuators which pump air into the controllable gas bearing in the vertical direction, $\mathbf{c}$ are the piezo actuators which pump air into the controllable gas bearing in the horizontal direction, $\mathbf{d}$ is the flexible shaft, $\mathbf{e}$ is the sensor measuring the vertical displacement of the disc, $\mathbf{f}$ is the disc and $\mathbf{g}$ is the sensor measuring the horizontal displacement of the disc.

$$
y=C x+D u
$$

Where:

$$
G=\left[\begin{array}{l|l}
A & B \\
\hline C & D
\end{array}\right]
$$

As shown in [10] it is possible to state a model structure as given in Eq . (4) - (7) using a standard state space description of the system as given in equation (2).

$$
\begin{aligned}
\mathbf{A}_{6 \times 6} & =\left[\begin{array}{ccc}
0 & \mathbf{I} & 0 \\
\mathcal{K} & \mathcal{D} & \mathcal{B} \\
0 & 0 & -\mathcal{P}
\end{array}\right] \\
\mathbf{B}_{6 \times 2} & =\left[\begin{array}{l}
0 \\
0 \\
\mathcal{P}
\end{array}\right] \\
\mathbf{C}_{2 \times 6} & =\left[\begin{array}{lll}
\mathbf{I} & 0 & 0
\end{array}\right] \\
\mathbf{D}_{2 \times 2} & =\left[\begin{array}{ll}
0
\end{array}\right]
\end{aligned}
$$

Here $\mathcal{K}$ is the stiffness matrix of the system and is in $\left[\frac{N}{k g \mu m}\right], \mathcal{D}$ is the damping matrix of the system expressed in $\left[\frac{\mathrm{Ns}}{\mathrm{kg} \mu \mathrm{m}}\right]$ and $\mathcal{B}$ is the input gain matrix in $\left[\frac{\mathrm{N}}{\mathrm{kgV}}\right]$. The matrix $\mathcal{P}$ is designed as a diagonal matrix with each element $p_{j}$ defined by equation (8) as a low pass filter describing the actuator dynamics, i.e linear displacement of piezo actuators as a function of the input voltage:

$$
h_{j}(s)=\frac{p_{j}}{s+p_{j}} \quad j \in\{1,2\}
$$

$h_{j}$ is the first order low pass filter through which the system is actuated.

The model parameters have been identified using a grey box open loop prediction error method. The parameters are given in Table 1 for the gas bearing without rotation and in Table 2 when the shaft is rotating at 1100 RPM. A rotational speed of 1100 RPM has been chosen as a prove of concept to show that it is possible to diagnose faults during rotation. 


$$
\begin{aligned}
\mathcal{K} & =\left[\begin{array}{cc}
-1.79 \cdot 10^{6} & 1.726 \cdot 10^{4} \\
1.061 \cdot 10^{4} & -1.926 \cdot 10^{6}
\end{array}\right] \\
\mathcal{D} & =\left[\begin{array}{ll}
-283.2 & -13.82 \\
-8.152 & -219.7
\end{array}\right] \\
\mathcal{B} & =\left[\begin{array}{cc}
4.318 \cdot 10^{6} & -2.672 \cdot 10^{5} \\
9.204 \cdot 10^{4} & 4.81 \cdot 10^{6}
\end{array}\right] \\
\mathcal{P} & =\left[\begin{array}{cc}
1021 & 0 \\
0 & 944.5
\end{array}\right]
\end{aligned}
$$

Table 1. Table of the identified parameters for the model of the active gas bearing when the shaft is not rotating.

$$
\begin{aligned}
\mathcal{K} & =\left[\begin{array}{cc}
-1.79 \cdot 10^{6} & 3.43 \cdot 10^{4} \\
3.171 \cdot 10^{4} & -1.931 \cdot 10^{6}
\end{array}\right] \\
\mathcal{D} & =\left[\begin{array}{cc}
-241.6 & 45.97 \\
7.94 & 212.1
\end{array}\right] \\
\mathcal{B} & =\left[\begin{array}{cc}
-4.446 \cdot 10^{6} & 7887 \cdot 10^{4} \\
6085 & 4.853 \cdot 10^{6}
\end{array}\right] \\
\mathcal{P} & =\left[\begin{array}{cc}
898.9 & 0 \\
0 & 955.7
\end{array}\right]
\end{aligned}
$$

Table 2. Table of the identified parameters for the model of the active gas bearing when the shaft is rotating with 1100 RPM.

The identified model is discretised using the zero order hold method for design and implementation of controllers on the active gas bearing. A sampling rate of 5000 $\mathrm{Hz}$ (sampling time $=0.0002 \mathrm{~s}$ ) is chosen for the discrete model.

\section{CONTROLLER SETUP}

Two different controllers are discussed and implemented to examine the effect of different control architectures in connection with fault diagnosis of the active gas bearings. The first controller implemented is a simple P-controller where the gain is determined by examining its root, while the second controller is an observer based LQR controller.

\subsection{Design of $\mathrm{P}$ controller}

In order to be able to examine the root of the closed loop system poles the controller is given as in equation (9).

$$
K=k\left[\begin{array}{ll}
1 & 0 \\
0 & 1
\end{array}\right]
$$

Using same control in both directions is found appropriate due to the similar dynamics for the two directions. This description of the controller with $k$ being a scalar makes it possible to follow the loci of the poles where as for a proper MIMO controller, with non-diagonal elements different from zero, the Gershgorin circle theorem would be applicable instead. The root locus plot is shown on Figure 3 for the control design given in equation (9).

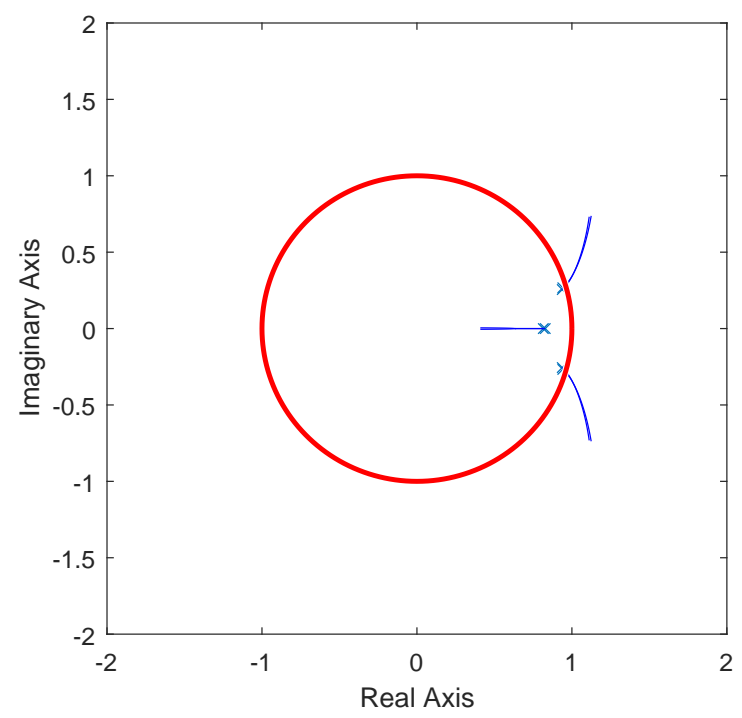

Fig. 3. Root locus plot of the rotor gas bearing a proportional gain between 0 and -10 . The system is found to be stable for a gain from each position error to each input of 0.2 or smaller.

Using Figure 3 the controller gain $\mathrm{k}$ was chosen to be -0.2 and the final controller is thus given in equation (10).

$$
K=\left[\begin{array}{cc}
-0.2 & 0 \\
0 & -0.2
\end{array}\right][V / \mu m]
$$

The controller design was chosen to examine the effect of a simple controller without considering any cross coupling.

\subsection{Design of LQR controller}

The second controller used for experiments is a $L Q R$ controller designed using a full order observer. The observer gain is shown in equation (11) and the state feedback gain is given in equation (12). 


$$
\begin{aligned}
\mathbf{L}= & {\left[\begin{array}{cc}
-0.17 & 0.00 \\
0.00 & -0.19 \\
-3.99 & 1.94 \\
2.62 & -15.77 \\
-0.02 & -0.00 \\
0.00 & -0.02
\end{array}\right] } \\
\mathbf{F}= & {\left[\begin{array}{cc}
-0.08 & 2.36 \\
2.5 & 0.10 \\
0.00 & -0.00 \\
-0.00 & -0.00 \\
0.04 & -5.16 \\
-5.70 & 0.04
\end{array}\right] }
\end{aligned}
$$

\subsection{Controller Implementation}

With both a proportional and observer based control design the detectors are implemented using the active fault diagnosis scheme as presented on Figure 4.

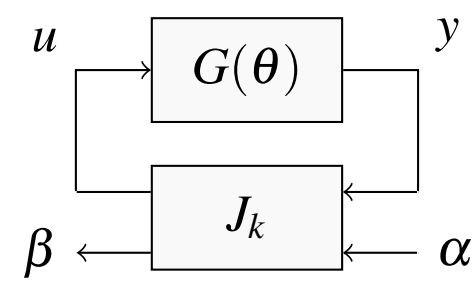

Fig. 4. Experimental test setup. $G(\theta)$ represents the gas bearing, $J_{k}$ is the nominal controller augmented with the two signals $\alpha$ which is the excitation signal and $\beta$ which is the residual signal.

Here $J_{k}$ is the controller augmented with the vector signals $(\alpha, \beta)$ and $\theta$ denotes the parametric faults in the system. The noise has been omitted from Figure 4 and will be considered in section 6.. For an observer based controller design the setup in Figure 4 is realised by letting the signal $\beta$ be the innovation signal and by letting $\alpha$ be a control signal of $J_{k}$, see Figure 4. With the controller architecture from Figure 4 the detection signal $\beta$ is given by equation (13).

$$
\beta=\left[\begin{array}{l}
\beta_{h} \\
\beta_{v}
\end{array}\right]=S(\theta) \alpha=S(\theta)\left[\begin{array}{l}
\alpha_{h} \\
\alpha_{v}
\end{array}\right]
$$

Here $S(\theta)$ is the fault signature system. $\beta_{h}$ is the residual in the horizontal direction and $\beta_{v}$ is the residual in the vertical direction. Equivalently $\alpha_{h}$ is the excitation signal in the horizontal direction and $\alpha_{v}$ is the excitation signal in the vertical direction. The fault signature system has the property of being zero in the fault free case and non-zero when a fault has occurred or as written in equation (14).

$$
S(\theta)=\left.0\right|_{\theta=0}
$$

It is always possible to design an augmented controller scheme as in Figure 4 by a coprime factorisation of controller and plant. The method is explained in the supplemental material where it is shown how to design $J_{k}$ and find the Youla-Kucera factorisation given different controller designs.

The ability of the gas bearing to suppress disturbances is shown on Figure 5 for both the nominal gas bearing and for each of the two implemented controllers. The plot shows that both controllers give the system increased disturbance rejection compared to the open loop system.
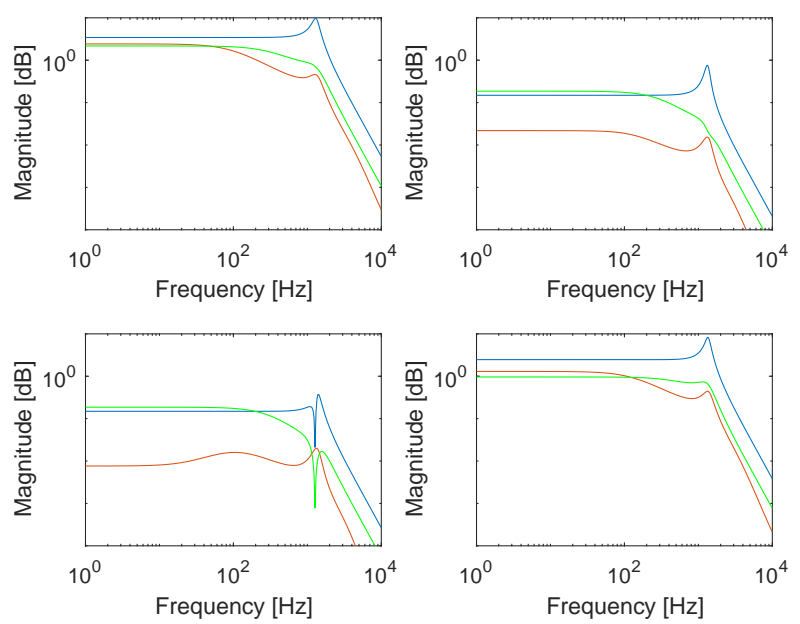

Fig. 5. Plot of the gain from a displacement disturbance to the displacement of the rotor for when the rotor is not rotating. The left column is for a disturbance in the horizontal direction while the right column is for a disturbance in the vertical direction. Equivalently the top row is for the position of the rotor in the horizontal direction while the bottom row is for the position in the vertical direction. The blue line represent the gas bearing without any control, the red line represent the gas bearing with the LQR controller implemented and the green line represent the gas bearing with the proportional controller implemented.

\section{FAULT DESCRIPTION}

In this section the possible faults considered are discussed and the fault signature system is derived based on the faults to be detected. Typically faults on an active gas bearing are due to wear of the actuators and defective sensors. The model structure established in section 2. forms the basis for the fault structure introduced in this section. 
In this paper the faults considered are represented as parametric faults. Faults on the actuators are represented as a decrease in the input gain, while a fault on a sensor is modelled as a decrease in the sensor readings. The different possible faults have been summarised in Table 3 together with the bounds associated with each of the faults. With the bounds as shown in Table 3 , the fault free case corresponds to $\theta_{i}=0$ where as a complete failure hence a fault of $100 \%$ corresponds to $\theta_{i}=-1$ where $i$ represent the specific fault.

The possible faults are described using a linear fractional transformation as shown in equation (15).

$$
G(\theta)=\mathcal{F}_{u}\left(G_{a u g}, \theta\right)
$$

Further, the connection between $z$ and $w$ is given by equation (16).

$$
w=\theta z
$$

Where $\theta$ is a diagonal matrix with the four faults considered, i.e.

$$
\theta=\left[\begin{array}{cccc}
\theta_{1} & 0 & 0 & 0 \\
0 & \theta_{2} & 0 & 0 \\
0 & 0 & \theta_{3} & 0 \\
0 & 0 & 0 & \theta_{4}
\end{array}\right]
$$

Where the augmented system is defined as in equation (18).

$$
G_{a u g}=\left[\begin{array}{ll}
G_{z w} & G_{z u} \\
G_{y w} & G_{y u}
\end{array}\right]
$$

The augmented plant $G_{a u g}$ is shown in equation (19).

$$
G_{\text {aug }}=\left[\begin{array}{ccc|ccc}
0 & \mathbf{I} & 0 & 0 & 0 & 0 \\
\mathcal{K} & \mathcal{D} & \mathcal{B} & 0 & 0 & 0 \\
0 & 0 & -\mathcal{P} & \mathcal{P} & 0 & \mathcal{P} \\
\hline \mathbf{I} & 0 & 0 & 0 & 0 & \mathbf{I} \\
0 & 0 & 0 & \mathbf{I} & 0 & 0 \\
\mathbf{I} & 0 & 0 & 0 & 0 & 0
\end{array}\right]
$$

\section{DESIGN OF THE EXCITATION SIGNAL}

During fault diagnosis, the process is disturbed by the excitation signal. Reducing the disturbance to the production is imperative so that the performance degradation is minimised during fault diagnosis. It is therefore convenient to use an excitation signal that makes it possible to detect the faults while trying not to disturb the process. A method for determining the frequency of the sinusoidal excitation signal was introduced in [24]. The goal of the method is to maximise the impact of faults on the fault signature system while minimising the impact of the excitation signal on the outputs.

Based on Fig. 4 the transfer function from the excitation signal to the residual signal is found. It is thus possible to determine the impact of the faults on the residual signal. It is worth noting that the transfer function from the excitation signal to the residual signal depends on the specific fault occurring and the known nominal model as well as controller parameters. Equivalently the transfer function from the excitation signal to the outputs of the system is required to define an efficiency criteria:

$$
f_{\alpha}=\frac{\xi_{r}(\omega)}{\xi_{y}(\omega)}
$$

Here $\xi_{r}(\omega)$ is the frequency dependent gain from the excitation signal $\alpha$ to the output of the fault signature system $\beta$. Equally $\xi_{y}(\omega)$ is the frequency dependent highest singular value for the single input multiple output system from the excitation signal $\alpha$ to the outputs $y$. With $f_{\alpha}$ defined in equation (20) the frequency of the excitation signal can be determined as shown in equation (21).

$$
\omega_{\alpha}=\underset{\omega_{1} \leq \omega \leq \omega_{2}}{\operatorname{argmax}} f_{\alpha}(\omega)
$$

Here $\omega_{1}$ and $\omega_{2}$ are chosen as the bound for the frequencies considered using for the excitation signal. The active gas bearing has two possible inputs for the excitation signal and two possible residual signals where four different combinations are possible and should be considered. A plot of the efficiency gain of the residual for a fault corresponding to a gain degradation of $50 \%$ for each of the four faults, using the proportional controller is shown on Figure 6 a to Figure $6 \mathrm{~d}$ where the four different possible excitation and residual signal combinations have been tested.

From Figure 6 a to Figure $6 \mathrm{~d}$ it is seen that $f_{\alpha}$ increases as the frequency of the excitation signal is increased. The frequency of the excitation signal was found using equation (21) with $\omega_{1}=1 \mathrm{rad} / \mathrm{s}$ and $\omega_{2}=62 \mathrm{rad} / \mathrm{s}$. The excitation has been chosen to be $62 \mathrm{rad} / \mathrm{s}$, such that the excitation signal is kept smooth given the sampling rate. With the frequency of the excitation signal selected it is necessary to examine the phase shift of the residual signal given the fault signature system for each of the 4 different parametric faults. It is possible to calculate the phase shift for the interval, fault free to failure, given the chosen excitation signal. The phase shift versus the magnitude of the faults is shown on Figure 7 using the excitation signal $\alpha_{h}$ and residual signal $\beta_{v}$. 


\begin{tabular}{|l|l|l|l|}
\hline Fault description & Notation & Bound & \begin{tabular}{l} 
Description \\
\hline Horizontal actuator
\end{tabular}$\theta_{1}$ \\
Vertical actuator & $\theta_{2}$ & {$\left[\begin{array}{l}{[0-1]} \\
\theta_{3}\end{array}\right.$} & $\begin{array}{l}\text { The fault on the opening degree of the horizontal actuator is } 100 \cdot\left(-\theta_{1}\right) \% \\
\text { is expected to be. This results in a reduction of the horizontal input gain. } \\
\text { The fault on the opening degree of the vertical actuator is } 100 \cdot\left(-\theta_{2}\right) \% \\
\text { is expected to be. This results in a reduction of the vertical input gain. } \\
\text { The fault on the measured distance of the horizontal displacement sensor is } \\
100 \cdot\left(-\theta_{3}\right) \% . \text { This is equivalent to a reduction in the } \\
\text { horizontal output gain and the controller is then demanding wrong actuation. } \\
\text { The fault on the measured distance of the vertical displacement sensor is } \\
100 \cdot\left(-\theta_{4}\right) \% . \text { This is equivalent to a reduction in the vertical output } \\
\text { gain and the controller is then demanding wrong actuation. }\end{array}$ \\
\hline
\end{tabular}

Table 3. Lookup table for the different possible faults

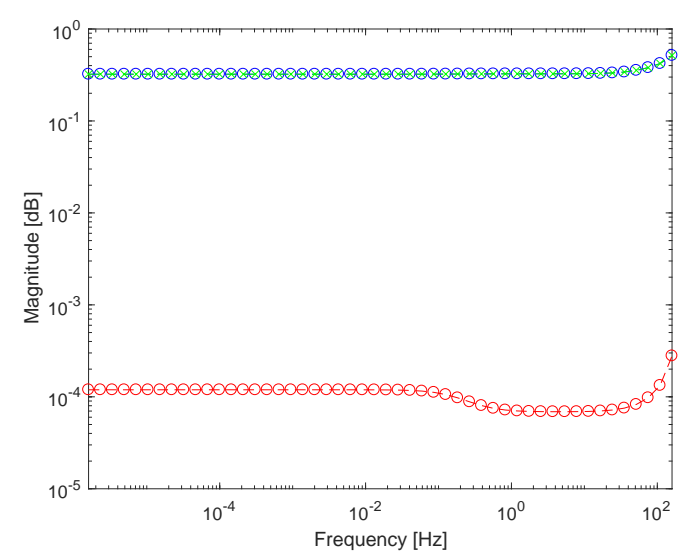

(a)

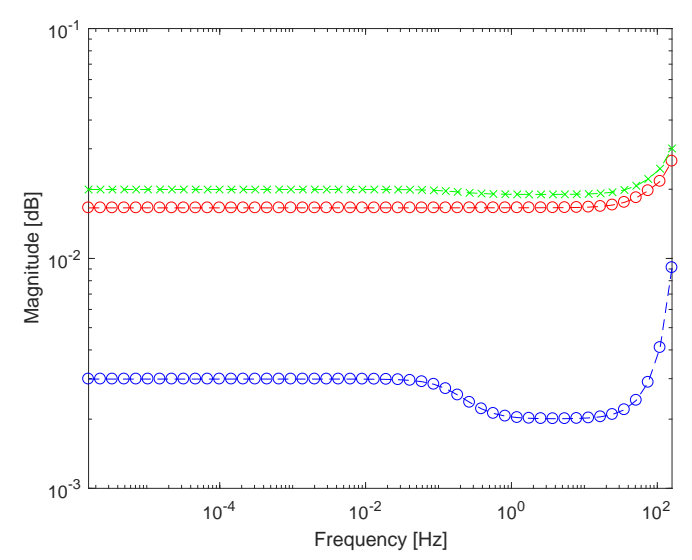

(c)

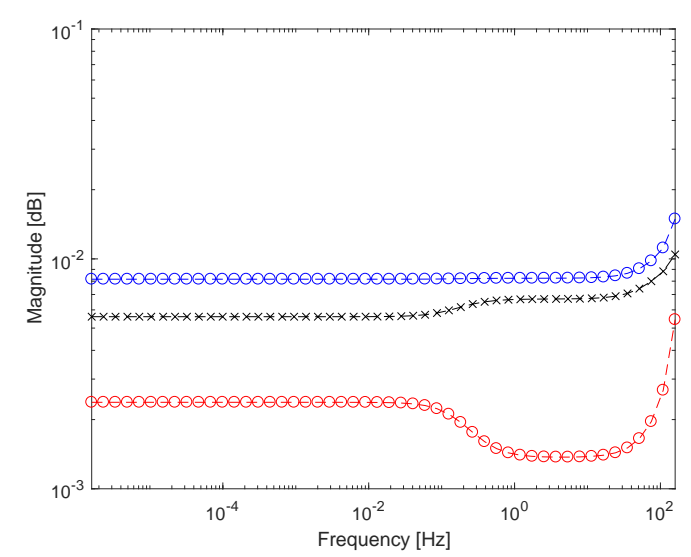

(b)

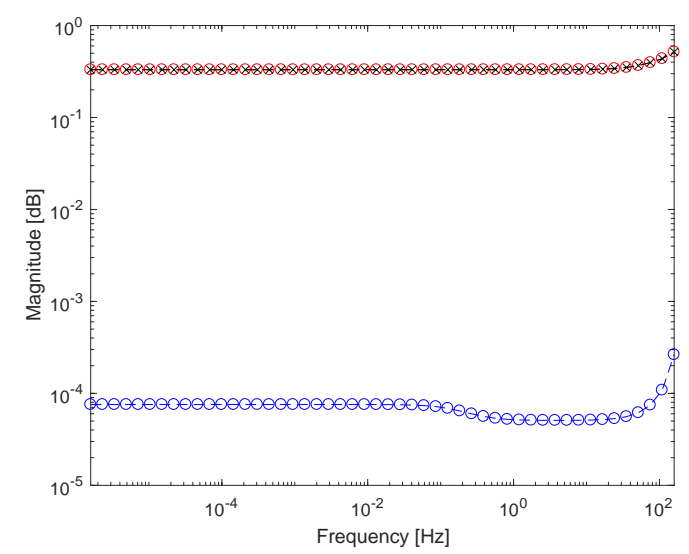

(d)

Fig. 6. Plot of $f_{\alpha}$ versus the frequency of the excitation signal where $\alpha_{h}$ and $\beta_{h}$ is used in Figure 6a, $\alpha_{h}$ and $\beta_{v}$ in Figure $6 \mathrm{~b}, \alpha_{v}$ and $\beta_{h}$ in Figure $6 \mathrm{c}$ and $\alpha_{v}$ and $\beta_{v}$ in Figure $6 \mathrm{~d}$. The blue line represent $f_{\alpha}$ given a fault on the horizontal actuator, the red line is for a fault on the vertical actuator, the green line represent the horizontal sensor and the black line represent the vertical sensor. Faults on the horizontal sensor cannot be detected when using $\beta_{v}$ and faults on the vertical sensor cannot be detected when using $\beta_{h}$. 


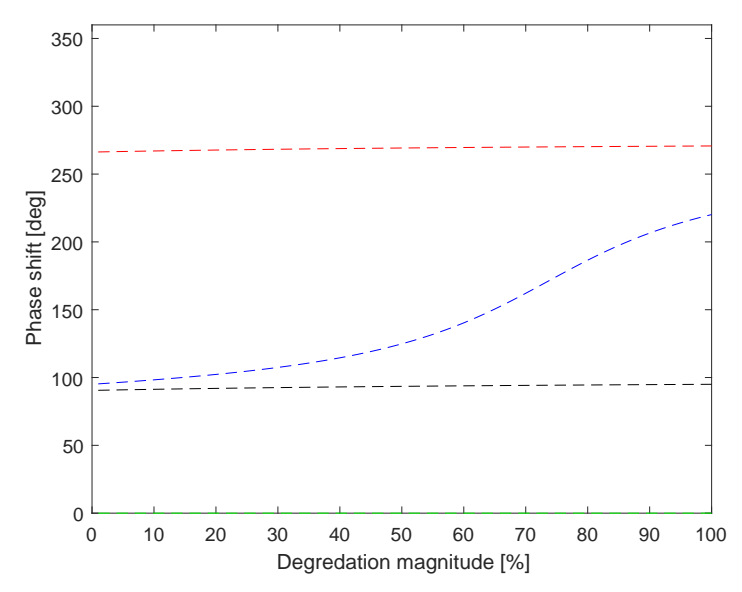

Fig. 7. Plot of the phase shift versus the magnitude of each of the 4 faults considered using the horizontal $\alpha$ signal and vertical $\beta$ signal. The blue line represents a fault on the horizontal actuator, the red line is for a fault on the vertical actuator, the green is for a fault on the horizontal sensor and the black represents the vertical sensor.

Based on Figure 7 the phase shift given each of the four possible faults is found with an excitation signal frequency of $62 \mathrm{rad} / \mathrm{s}$ when using the excitation signal $\alpha_{h}$ and residual signal $\beta_{v}$. It is possible to make similar plots given the other combinations of excitation and residual signals.

It is not possible to detect faults on the horizontal displacement sensor using the horizontal residual as seen from Figure $6 \mathrm{~b}$ and $6 \mathrm{~d}$. Equivalently it is found impossible to detect faults on the vertical displacement sensor using the vertical residual signal as seen on Figure 6a and 6c. It is therefore found necessary to use both the vertical and horizontal residual signals to be able to detect all the predetermined possible faults.

\section{DETECTOR DESIGN}

A detector design is required in order to detect and isolate faults. It is decided in this paper to use a matched filter detector design. Such a matched filter detector design was introduced in [26] and it was shown in [24] how to apply it to the case of active fault detection based on the fault signature system. Based on Figure 8, diagnosis is based on equation (22) where the disturbance is introduced.

$$
\beta=S \alpha+d
$$

The signal $\beta$ is as shown in equation (23) used as the residual signal denoted $r$ in keeping with common practice, i.e

$$
r=\beta
$$

Here the disturbance $d$ is assumed to be white gaussian noise (WGN). Based on the results in [26] it is possible to formulate a $\mathcal{H}_{0}$ hypothesis as in equation (24) and a $\mathcal{H}_{1}$ hypothesis as in equation (25).

$$
\begin{aligned}
& \mathcal{H}_{0}: r[n]=d[n] \\
& \mathcal{H}_{1}: r[n]=|S(\theta)| \alpha\left[n-n_{\phi}\right]+d[n]
\end{aligned}
$$

Here $r[n]$ is the fault signature signal as given in equation (23), $\alpha$ is the excitation signal and $|S(\theta)|$ is the gain of the fault signature system given the selected excitation frequency and $n_{\phi}$ is the delay of the excitation signal though the fault signature system. The fault free case thus results in simply white noise, while the faulty case results in a known signal with an unknown delay and unknown amplitude. The unknown delay and amplitude both depend on the specific fault occurring and the magnitude of the fault. Identifying the delay and amplitude thus make it possible to isolate the faults. A correlator design as presented in equation (26) is implemented to determine which fault most likely occurred.

$$
n_{\phi}=\arg \max _{n_{0} \in \tau}\left(\sum_{n=n_{0}}^{n_{0}+m-1} r[n] \alpha\left[n-n_{0}\right]\right)
$$

Here $n_{\phi}$ is the identified delay of the fault signature signal through the fault signature system, $m$ is the length of the matched filter window and $\tau$ is the set of possible delays considered. With the delay identified a test statistic can be generated using equation (27).

$$
T[n]=\sum_{n=0}^{m-1} r[n] \alpha\left[n-n_{\phi}\right]
$$

A schematic of the detector design explored in this section is shown in Figure 8.

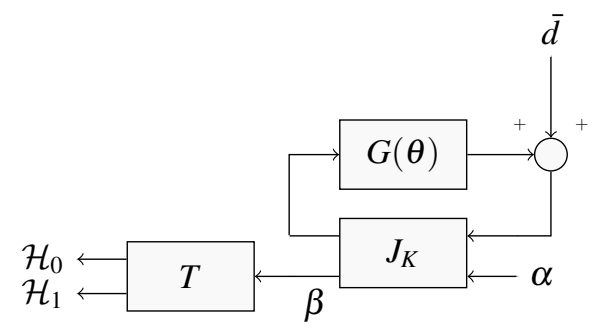

Fig. 8. Experimental test setup. $G(\theta)$ represents the gas bearing, $J_{k}$ is the augmented controller, $\alpha$ is the excitation signal, $\beta$ is the residual signal. The block $T$ is the statistical detector implemented.

Based on this test statistic it is possible to determine the threshold $(\gamma)$ and probability of detection $\left(P_{D}\right)$. It is in 
this paper decided to base the threshold on the probability of a false alarm $\left(P_{F A}\right)$. Before the threshold can be determined it is furthermore required to quantify the detector signals energy. In equation (28) the energy of the detector is given.

$$
\varepsilon=m \frac{A_{|S(\theta)|}}{2}
$$

Here $A_{|S(\theta)|}$ is the amplitude of the fault signature signal. Since more than one fault is considered $A_{|S(\theta)|}$ is chosen to be the highest amplitude of the fault signature signal given the faults and fault magnitudes considered possible. With the energy of the signal determine it is possible to decide on a threshold for the test statistic given a probability of false alarm as shown in equation (29).

$$
\gamma=\sqrt{\sigma^{2} \varepsilon} Q^{-1}\left(P_{F A}\right)
$$

Here $\sigma^{2}$ is the variance of the measurement noise and $Q(*)$ is the complementary cumulative distribution function. With the use of the of the determined probability of a false alarm the probability of detection can be calculated using equation (30).

$$
P_{D}=Q\left(Q^{-1}\left(P_{F A}\right)-\sqrt{\mathcal{D}}\right)
$$

Here $\mathcal{D}$ is given in equation (31) and is the deflection coefficient which is based on the expected value of the test statistic for the case of a fault and no fault together with the variance of the test statistic in the fault free case.

$$
\mathcal{D}^{2}=\frac{\left(E\left(T ;\left.\mathcal{H}_{1}\right|_{F_{k}}\right)-E\left(T ; \mathcal{H}_{0}\right)\right)^{2}}{\operatorname{var}\left(T ; H_{0}\right)}
$$

$F_{k}$ is the notation for which of the considered faults that has occurred, thus the deflection coefficient and hence the probability of the detection changes depending on which fault that is considered.

\subsection{Design and Performance of the Whitening Filter}

The residual signal used in [21] and here denoted as $\beta$ is filtering a systems noise signals through a linear filter. The detector design is based on the noise in equation (24) being white. This will in general not be satisfied when using the $\beta$ signal as the residual for fault detection on the active gas bearing. A whitening filter is therefore needed.

The main contributor to noise $(d)$ on the active gas bearing is identified to be the displacement sensors and a open loop experiment without any excitation was conducted which showed the noise to be approximately Gaussian and white. Using the signal $\beta$ as the residual signal results in that the measurement noise is subject to a linear filter. It was proposed in [24] to design a whitening filter for the fault free case. Since the active gas bearing is open loop stable it is possible to find a whitening filter that also keep the variance of the noise the same on the residual signal as on the measurements. The residual signal is therefore reformulated in equation (32) using the known signal $\beta$ and a linear whitening filter $W$.

$$
r=W \beta
$$

The whitening filter $W$ is designed such that the measurement noise on the active gas bearing is white in the fault free case on the residual signal. A throughout description of how to design the whitening filter can be found in [24]. A block diagram where the filter is implemented and the identified source of the noise is shown is shown in Figure 9.

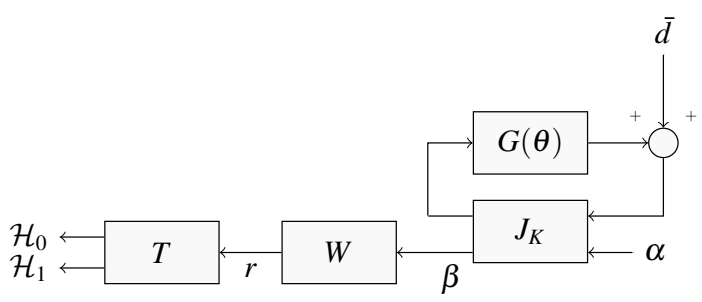

Fig. 9. Experimental test setup. $G(\theta)$ represents the gas bearing, $J_{k}$ the augmented controller, $\alpha$ is the excitation signal, $\beta$ is the residual signal subject to non-white noise, $W$ is the whitening filter and $r$ is the residual signal subject to white noise.

\section{EXPERIMENTS}

Based on the possible faults described in section 4., four experiments are conducted in order to show that it is possible to detect and isolate each of the four different faults considered using a one excitation signal and one residual signal. Each of the faults considered are induced as $\theta_{i}=-0.5$. Even though the faults are quite severe a gain reduction of $50 \%$ on the actuators is found to be likely. Such a reduction can be due to pressure loss or something getting stuck in the tube letting air into the active gas bearing. Due to the severity of the faults a linearised version of the fault signature system cannot be considered for the fault diagnosis.

\subsection{Analysis of the noise on the Gas bearing}

Firstly the effect of the whitening filter is examined. In order to examine the effect of the whitening filter $W$ a residual signal has been obtained for the fault free gas bearing without any excitation signal applied. On Figure 10 the normalised autocorrelation is shown using the signal $\beta$ which is the residual signal before any whitening has been conducted. 

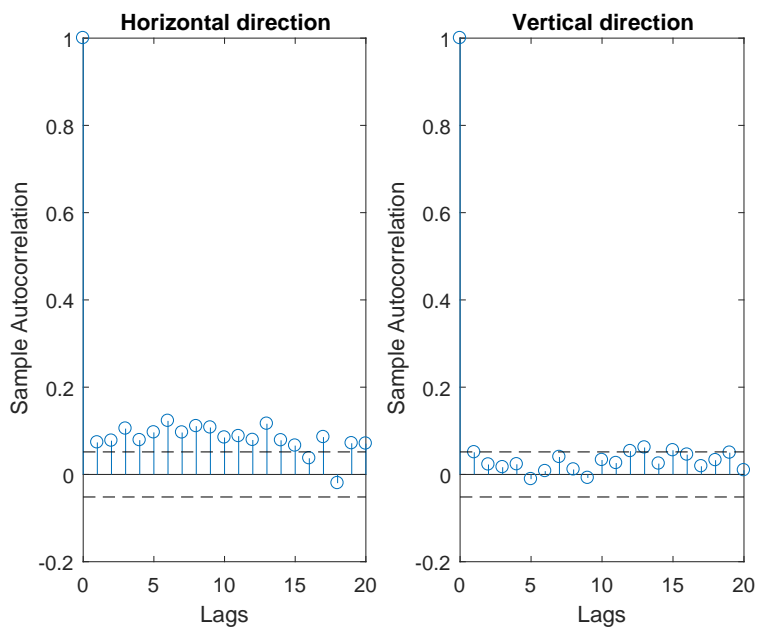

Fig. 10. Plot of the autocorrelation of $\beta$ in the fault free case. The stems show the correlation depending on the number of lags, while the black stippled lines are the upper and lower confidence bounds.

It is clear from Figure 10 that the signal is not white even though the noise the system originally was subject to is. Equivalently the same test can be conducted using equation (32) as the fault signature system. In this case the normalised autocorrelation is shown on Figure 11.
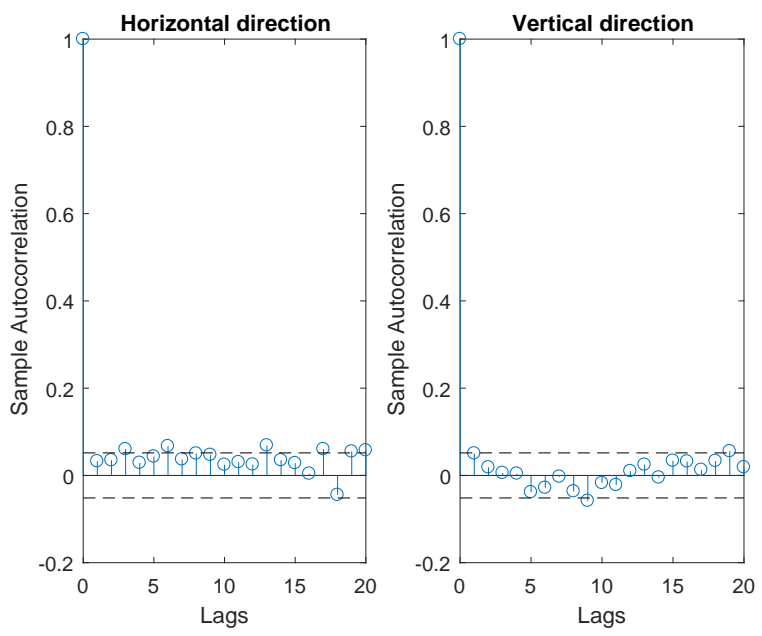

Fig. 11. Plot of the autocorrelation of $r$ in the fault free case. The stems show the correlation depending on the number of lags, while the black stippled lines are the upper and lower confidence bounds.

From Figure 11 it is seen that the residual signal is much closer to being white in the fault free case. For a perfect model this experiment could be conducted with an active excitation signal applied, however even though the model is able to produce a good fit it is not considered perfect and the excitation signal would thus pollute the experi- ment. This imperfection of the model is also possible to see in that the residual signal, even though it is closer to being white, it is still not within the bounds of the statistical uncertainty for the autocorrelation function.

With the whitening filter verified an analysis of the noise on the residual signal is required. The residual signal has been monitored without any excitation signal applied and a normalised histogram is shown on Figure 12 for the horizontal direction and vertical direction.
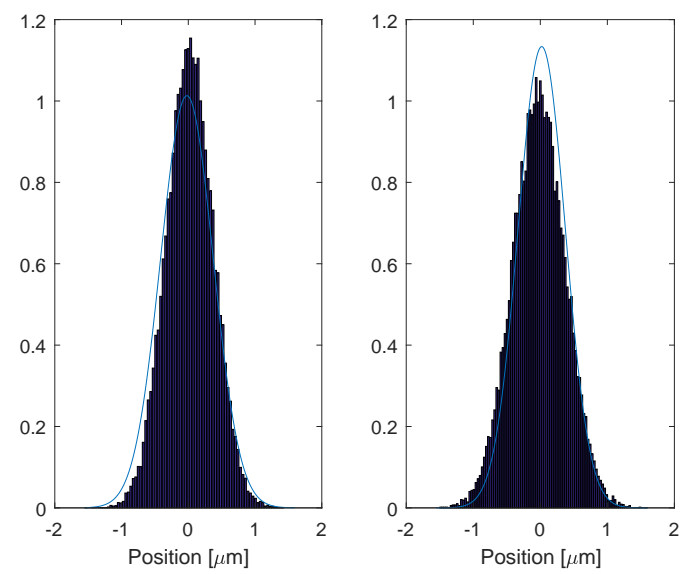

Fig. 12. Histogram of the signal $r$ in the fault free case with the horizontal direction on the left hand side and the vertical direction on the right hand side.

It is clear from the two histograms that the residual signal is subject to approximately gaussian noise with a mean of zero in both directions. The variance was found in the horizontal direction to be 0.12 and in the vertical direction to be 0.15 .

\subsection{Diagnosing faults using a P-controller}

In the first experiment the residual signal from the horizontal direction is used whose variance the threshold is thus based upon. In this experiment the disc is not rotating. Before detection can be conducted it is needed to calculate the energy in the signal depending on the fault occurring and the magnitude of the possible fault. A plot of the gain through the fault signature system is shown on Figure 13. 


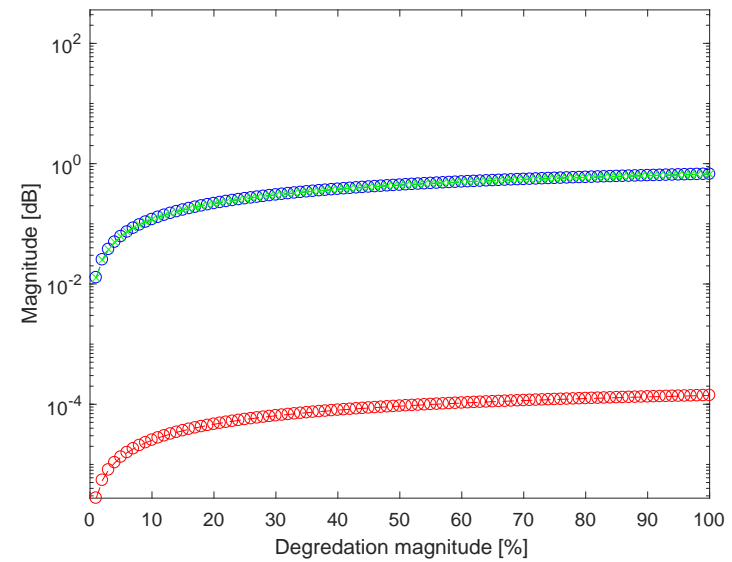

Fig. 13. Plot of the gain from the horizontal excitation signal $\alpha$ to the horizontal residual signal $r$ given each of the possible faults. The blue line represent a fault on the horizontal actuator, the red line is for a fault on the vertical actuator, the green is for a fault on the horizontal sensor and the black represent the vertical sensor.

From Figure 13 the gain through the fault signature system is found to be close to 1 for a fault on the horizontal piezo electric actuator of $50 \%$ and around 0.0003 for a fault on the vertical actuator of $50 \%$. A fault of $50 \%$ reduction in the opening degree was introduced on the horizontal actuator after 4 seconds. To determine whether it is possible to detect the fault a test statistic as shown in equation (27) is implemented. The threshold is determined using equation (29) as shown in equation (33).

$$
\begin{aligned}
\gamma & =\sqrt{\sigma^{2} \varepsilon} \cdot Q^{-1}\left(P_{F A}\right) \\
& =\sqrt{0.42^{2} \cdot 31275} \cdot Q^{-1}\left(2 \cdot 10^{-5}\right)=305
\end{aligned}
$$

The test statistic is then shown on Figure 14 for a fault on the horizontal actuator.

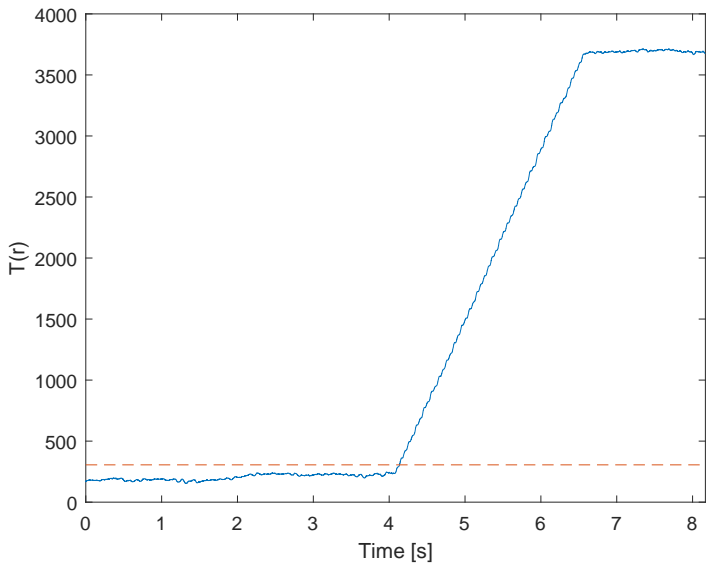

Fig. 14. Detector signal $T$ where a fault occurs after 4 seconds on the horizontal actuator. A window length of 2.5 seconds is used for the diagnosis.

It is clear from Figure 14 that it is possible to detect a fault on the horizontal actuator using the horizontal input and horizontal output. In order to determine which fault occur equation (26) is utilised. In order to determine the phase shift of the residual signal 4 different possible phase shifts are considered and are given in equation (34).

$$
\tau=[93,89,94,0]
$$

The phase shift set corresponds to the phase shift introduced given a $50 \%$ degradation $\left(\theta_{i}=-0.5\right)$ of each of the possible faults shown in Table 3 . The phase shift was found as the phase shift of the fault signature system $S(\theta)$ for each of the 4 different possible faults respectively. The result of the decision algorithm is shown on Figure 15 using equation (26).

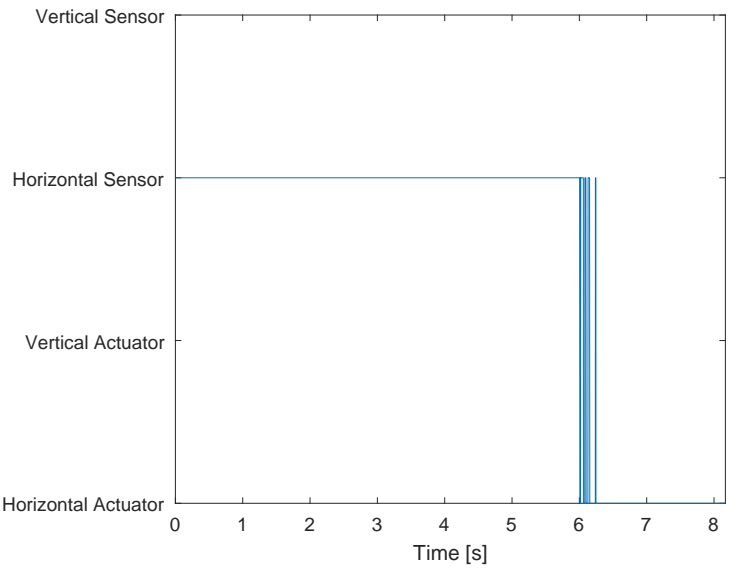

Fig. 15. Plot of the decision $n_{\phi}$ where a fault has been introduced on the horizontal actuator after $4 \mathrm{sec}$ onds. 
It is clear from Figure 15 that the fault is isolated almost 2 seconds after it has actually occurred and is detected. With a window of 2.5 seconds almost the whole window is used.

The decision algorithm determine a horizontal sensor fault to be most likely in the fault free case. There can be several reasons why the decision algorithm does not change between all the possible faults in the fault free case. The most obvious reason is because the model is not a perfect representation of the active gas bearings, thus the fault signature system is not zero in the fault free case. Furthermore the window length is chosen to be $2.5 \mathrm{sec}$ onds which makes small model deviations have a relative higher impact on the fault decision compared to the noise. This is clearly seen from Figure 16 where the window has been shortened to 0.2 seconds.

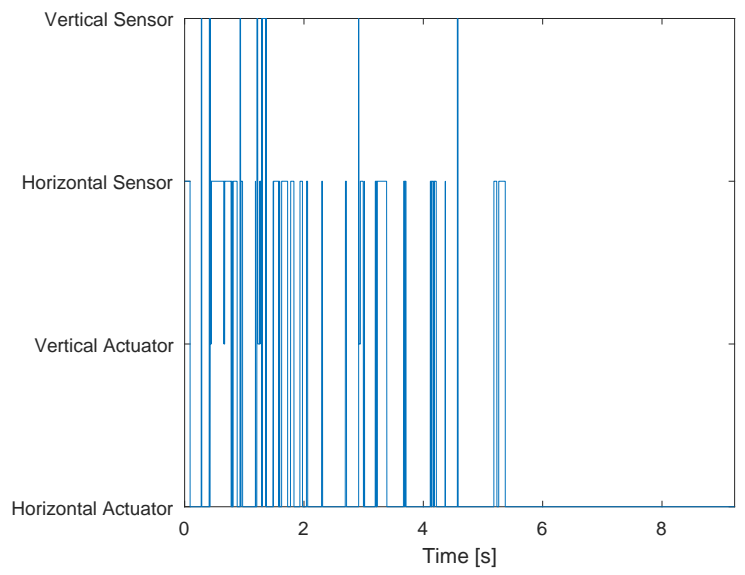

Fig. 16. Plot of the decision $n_{\phi}$ where a fault has been introduced on the horizontal actuator after $2.5 \mathrm{sec}$ onds. The window $m$ has here been reduced to 0.2 seconds.

From Figure 16 it is seen that the noise is dominant for the fault free case. Again the decision algorithm is able to isolate the correct fault, however in the fault free case the decision switches between all possible faults instead of being stuck at one as seen on Figure 15. The window length of 2.5 seconds was found unnecessary for detecting faults on the horizontal piezo actuator. However in the case of possible faults on the vertical actuator such a window size becomes relevant due to the much lower gain of the fault signature system in case of such a fault occurring. A fault of $50 \%$ degradation, is imposed on the vertical piezo actuator after 4 seconds and the detection signal $T(r)$ is shown on Figure 17. We recall that such a fault corresponds to the valve opening half the expected amount, hence the input gain of the system has been reduced.

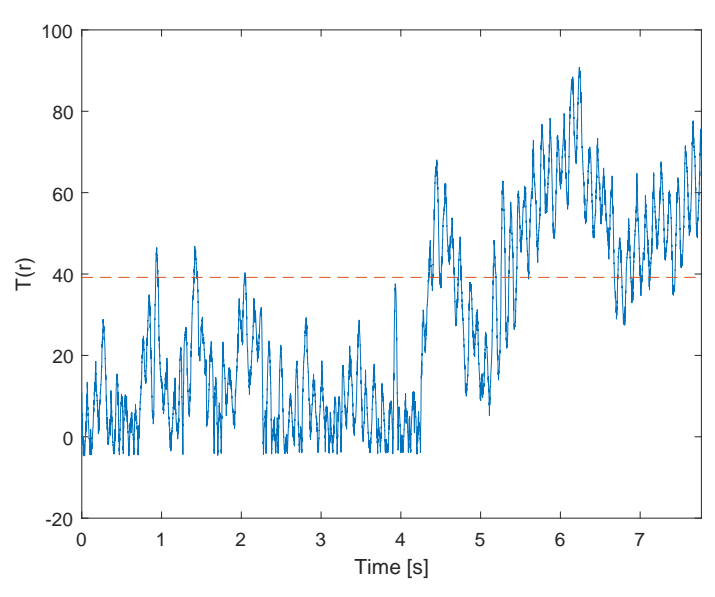

Fig. 17. Detector signal $T$ where a fault occurs after 4 seconds on the vertical actuator.

Recall that a false alarm occurs every time that the signal $T(r)$ goes above the stippled line before 4 seconds. We notice that we get more false alarms than we expected from the designed threshold. The unexpected amount of false alarms is assumed to be caused by the model uncertainties which has a higher impact on a vertical actuator fault than on the horizontal one in this experiment. This is due to the relative small gain through the fault signature system from the vertical excitation to the horizontal residual signal.

Again using equation (26) a decision on the possible fault occurring at each time step is shown on Figure 18.

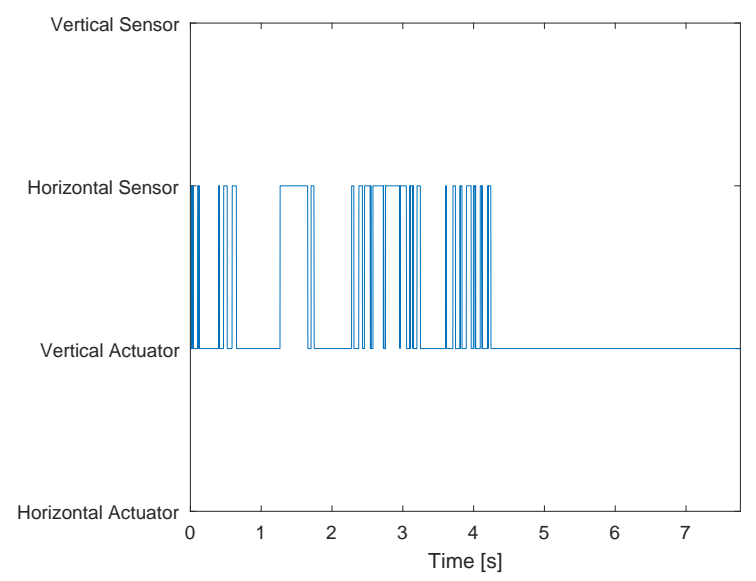

Fig. 18. Plot of the decision $n_{\phi}$ where a fault has been introduced on the vertical actuator after 4 seconds.

It is again found possible to isolate the fault which has been introduced to the active gas bearing using equation (26). 


\subsection{Diagnosing faults using cross coupling}

It has been shown possible to detect parametric actuator and sensor faults using a horizontal excitation and residual signal. It is in this experiment shown that it is possible to detect faults based on the cross coupling as well. Here it is done using a horizontal excitation signal and the vertical residual signal where again the disc is not rotating. As with the previous example the delay introduced to the residual signal given each of the possible parametric faults is found as shown in equation (35).

$$
\tau=[125,269,0,93]
$$

Experiments are conducted where each of the 4 possible parametric faults are introduced to the active gas bearing. A detection algorithm as presented in equation (27) is applied and the result for each of the 4 cases is shown in Figure 19.
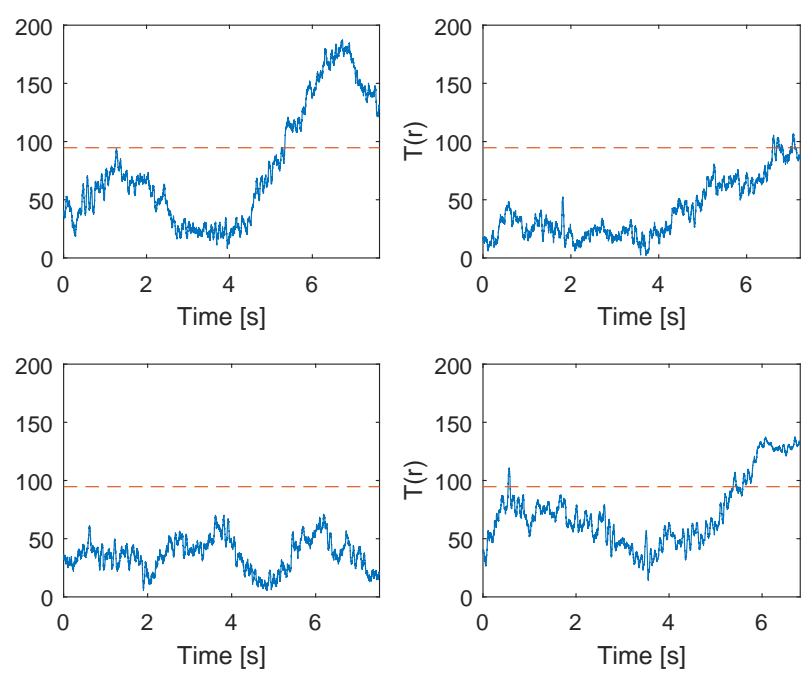

Fig. 19. Plot of $T[n]$ for the gas bearing when each of the 4 possible parametric faults is introduced respectively. A horizontal excitation signal is applied and the vertical output of the fault signature system is used for detection purposes. The top left plot is for a fault on the horizontal actuator where as the top right is for a fault on the vertical actuator. On the bottom left plot a fault is introduced on the horizontal sensor and on the bottom right a fault has been introduced on the vertical sensor instead.

It is seen form Figure 19 that by applying an excitation signal in the horizontal direction and using the vertical output of the fault signature system it is possible to detect faults on both the vertical and horizontal actuators. Where as it was expected that a fault on the horizontal actuator would not be detectable a fault on the vertical actuator should be. However the fault on the vertical displacement sensor is not detected due to the low gain from the excitation signal to the residual given a fault on the vertical displacement sensor.

\subsection{Detecting faults while the gas bearing is rotating.}

An experiment has been conducted where the shaft held in place by the active gas bearing has been set to rotate with a rotational speed of 1100 rounds per minute (RPM). The plant has been identified at a rotational speed of 1100 RPM and the plant has been diagnosed where a parametric fault of 50\% has been introduced on the horizontal actuator. A plot of the detector $(T[n])$ is shown on Figure 20.

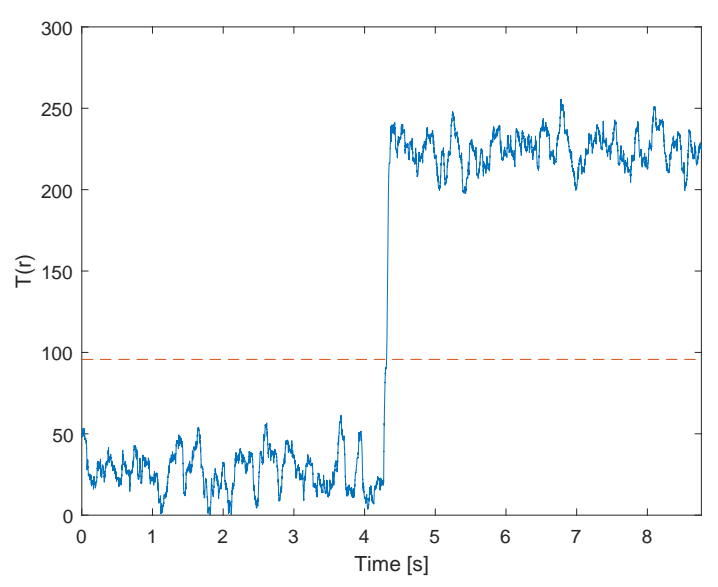

Fig. 20. Plot of $T[n]$ for the gas bearing when the shaft is rotating with 1100 RPM and a fault is introduced to the horizontal actuator. The excitation signal has been introduced in the horizontal direction and the horizontal output of the fault signature system has been used for the detection

The fault is introduced just after 4 seconds and a window length of 0.2 seconds is used. It is seen on Figure 20 that the fault is detected.

\subsection{Diagnosing faults using a LQR based controller}

For all the previous experiments the same proportional controller is used. An observer based controller is instead investigated here as described in section 3.. An excitation signal is used in the vertical direction and each of the 4 faults has been introduced to show the detectors ability to detect faults. The detection signal for each of the 4 faults being introduced is shown in Figure 21. 

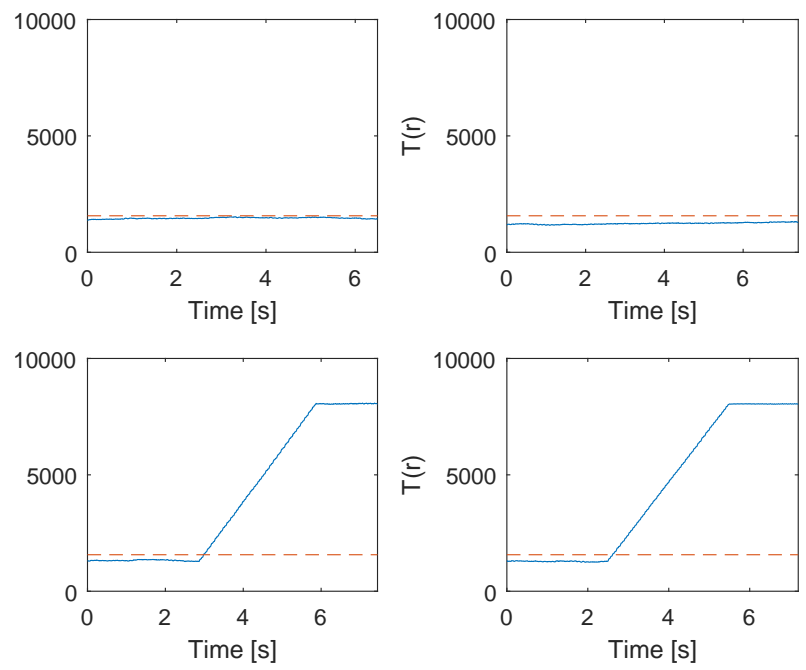

Fig. 21. Plot of $T[n]$ for the gas bearing when each of the 4 possible parametric fault is introduced respectively. A window length of 2.5 seconds is used. A vertical excitation signal is applied and the vertical output of the fault signature system is used for detection purposes. The top left plot is for a fault on the horizontal actuator where as the top right is for a fault on the vertical actuator. On the bottom left plot a fault is introduced on the horizontal sensor and on the bottom right a fault has been introduced on the vertical sensor instead.

It is seen from Figure 21 that it is still possible to detect different faults when the control scheme is changed. The method is thus shown able to apply with whichever linear control design used for the system. The isolation of the fault occurring is shown on Figure 22 for the fault on the vertical sensor.

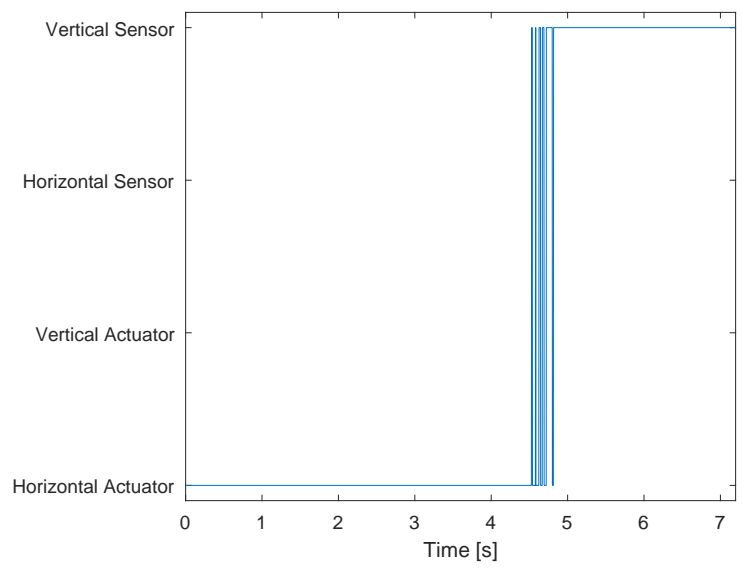

Fig. 22. Plot of the decision $n_{\phi}$ where a fault has been introduced on the vertical sensor after 3 seconds.
Again it is noted that a delay is present between a fault being detected and the isolation. The fault is proper isolated when the whole window is using data from after the fault has occurred.

\section{CONCLUSION}

It has been experimentally shown possible to detect and isolate different parametric faults on an active gas bearing using active fault diagnosis. It was shown advantageous to apply an active fault diagnosis method to an experimental active gas bearing test rig for diagnosis of parametric faults. The active fault diagnosis was based on the fault signature system. A maximum likelihood estimator was successfully applied to the problem. Two different controller architectures were tested, a proportional and an observer based, to show the methods flexibility. Both faults on the actuators and the sensors could be detected and isolated using the proposed control architectures. The results were achieved by the use of a single sinusoidal excitation signal found to minimize the disturbance on the system under the requirement of detectability of the considered faults. It was seen that using a single sinusoidal signal could make it difficult to distinguish between certain faults, but that the introduction of the excitation signal made redundant sensors not needed for isolation. The results thus showed consistency with the theory throughout the experiments. Furthermore threshold design with a whitening filter applied was shown to work. The assumptions used for the active fault diagnosis design was therefore found appropriate. The results have shown it possible to diagnose faults given a specific rotational speed of the active gas bearing. However as the rotational speed changes the dynamics of the active gas bearing is changing as well. It has been shown possible to control the system under such a setup using LPV control. The method should therefore in the future be extended for use with LPV controllers.

\section{Conflict of interest}

The authors declare no conflict of interest in preparing this article.

\section{Funding}

This research received no specific grant from any funding agency in the public, commercial, or not-for-profit sectors.

\section{REFERENCES}

[1] I. F. Santos, "On The Future Of Controllable Fluid Film Bearings," Mechanics \& Industry, vol. 12, no. 4, pp. 275281, 2011.

[2] B. T. Paulsen and S. Morosi and I. F. Santos, "Static, Dynamic, And Thermal Properties Of Compressible Fluid 
Film Journal Bearings," Tribology Transactions, vol. 54, no. 2, pp. 282-299, 2011.

[3] S. Morosi and I. F. Santos, "On The Modelling Of Hybrid Aerostatic-Gas Journal Bearings," Proceedings of the Institution of Mechanical Engineers, Part J: Journal of Engineering Tribology, vol. 225, no. 7, pp. 641-653, 2011.

[4] S. Morosi and I. F. Santos, "Active Lubrication Applied To Radial Gas Journal Bearings. Part 1: Modeling,” Tribology International, vol. 44, no. 12, pp. 1949-1958, 2011.

[5] S. Morosi and I. F. Santos, "Experimental Investigations Of Active Air Bearings," Proceedings of ASME turbo expo, vol. 7, pp. 901-910, 2012.

[6] F. G. Pierart and I. F. Santos, "Steady State Characteristics Of An Adjustable Hybrid Gas Bearing-Computational Fluid Dynamics, Modified Reynolds Equation and Experimental Validation," Tribology International, vol. 229, no. 7, pp. 807-822, 2015.

[7] F. G. Pierart and I. F. Santos, "Active Lubrication Applied To Radial Gas Journal Bearings. Part 2: Modelling Improvement And Experimental Validation," Tribology International, vol. 96, no. 7, pp. 237-246, 2016.

[8] F. G. Pierart and I. F. Santos, "Adjustable Hybrid Gas Bearing-Influence Of Piezoelectrically Adjusted Injection On Damping Factors And Natural Frequencies Of A Flexible Rotor Operating Under Critical Speeds," Journal of Engineering Tribology, vol. 230, no. 10, pp. 1209-1220, 2016.

[9] F. G. Pierart and I. F. Santos, "Lateral Vibration Control Of A Flexible Overcritical Rotor Via An Active Gas BearingTheoretical And Experimental Comparisons," Journal of Sound and Vibration, vol. 383, pp. 20-34, 2016.

[10] L. R. S. Theisen and H. H. Niemann and R. Galeazzi and I. F. Santos, "Enhancing Damping Of Gas Bearings Using Linear Parameter-Varying Control," Journal of Sound and Vibration, vol. 395, pp. 48-64, 2017.

[11] J. P. Amezquita-Sanchez and M. Valtierra-Rodriguez and D. Camarena-Martinez and D. Granados-Lieberman and R. J. Romero-Troncoso and A. Dominguez-Gonzalez, "Fractal Dimension-Based Approach For Detection Of Multiple Combined Faults On Induction Motors," Journal of Vibration and Control, vol. 22, no. 17, pp. 3638-3648, 2016.

[12] C. Lin and V. Makis, "Optimal Bayesian Maintenance Policy And Early Fault Detection For A Gearbox Operating Under Varying Load," Journal of Vibration and Control, vol. 22, no. 15, pp. 3312-3325, 2016.

[13] W. Moustafa and O. Cousinard and F. Bolaers and K. Sghir and J. P. Dron "Low Speed Bearings Fault Detection And Size Estimation Using Instantaneous Angular Speed," Journal of Vibration and Control, vol. 22, no. 15, pp. 34133425,2016

[14] I. Punčochár and M. Šmandl "On Infinite Horizon Active Fault Diagnosis For A Class Of Non-Linear Non-Gaussian Systems," International Journal of Applied Mathematics and Computer Science, vol. 24, no. 4, pp. 795-807, 2014.
[15] A. E. Ashari and R. Nikoukhah and S. L. Campbell "Active Robust Fault Detection In Closed-Loop Systems: Quadratic Optimization Approach," IEEE Transactions on Automatic Control, vol. 57, no. 10, pp. 2532-2544, 2012.

[16] J. K. Scott and R. Findeisen and R. D. Braatz and D. M. Raimondo, "Input Design For Guaranteed Fault Diagnosis Using Zonotopes," Automatica, vol. 50, no. 6, pp. 15801589,2014

[17] G. R. Marseglia and D. M. Raimondo, "Active Fault Diagnosis: A Multi-Parametric Approach," Automatica, vol. 79, pp. 223-230, 2017.

[18] S. M. Tabatabaeipour, "Active Fault Detection And Isolation Of Discrete-Time Linear Time-Varying Systems: A Set-Membership Approach," International Journal of Systems Science, vol. 46, no. 11, pp. 1917-1933, 2015.

[19] M. Šimandl and I. Punčochář, "Active Fault Detection And Control: Unified Formulation And Optimal Design," Automatica, vol. 45, no. 9, pp. 2052-2059, 2009.

[20] S. L. Campbell and R. Nikoukhah, "Auxiliary signal design for failure detection," Princeton University Press, 2015

[21] N. Poulsen and H. H. Niemann, "Active Fault Diagnosis Based On Stochastic Tests," International Journal of Applied Mathematics and Computer Science, vol. 18, no. 4, pp. 487-496, 2008.

[22] H. H. Niemann and N. Poulsen, "Active Fault Detection In MIMO Systems," American Control Conference, pp. 19751980, 2014.

[23] H. H. Niemann and N. Poulsen, "Estimation Of Parametric Fault In Closed-Loop Systems," American Control Conference, pp. 201-206, 2015.

[24] A. K. Sekunda and H. H. Niemann and N. K. Poulsen, "Detector Design For Active Fault Diagnosis In Closed-Loop Systems," International Journal of Adaptive Control and Signal Processing, vol. 32, no. 5, pp. 647-664, 2018.

[25] L. R. S. Theisen, "Advanced Control Of Active BearingsModelling, Design And Experiments," Technical University of Denmark, Department of Electrical Engineering, 2016.

[26] S. M. Kay, Fundamentals Of Statistical Signal Processing, Vol. II: Detection Theory, Prentice Hall, USA, 1998.

[27] H. H. Niemann, A YJBK Based Architecture For Fault Diagnosis And Fault-Tolerant Control, Linear System Theory, DTU publications, Denmark, 2015

[28] T. T. Tay and I. M. Y. Mareels and J. B. Moore, High Performance Control, Birkhauser, Chicago, 1998.

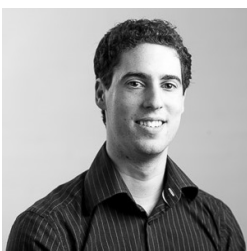

André Sekunda received the M.Sc degree in Control Engineering from Aalborg University in 2014 and his $\mathrm{PhD}$ degree from DTU in 2018. His research interests include Closed Loop Identification, Coprime Factorisations, and Active Fault Diagnostics. 


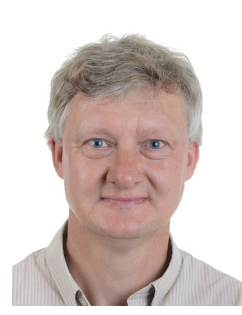

Hans Henrik Niemann received his M.Sc degree in mechanical engineering in 1986 and the Ph.D. degree in 1988 from Technical University of Denmark.

From 1988 to 1994 he had a research position and from 1994 he has been Ass. Professor in control engineering at Technical University of Denmark.

His research interests are: Optimal and robust control, fault detection and isolation, active fault diagnosis, fault tolerant control, controller architecture for controller switching and fault tolerant control, system and performance monitoring, controller anti-windup. He is first author of more than 80 journal and conferences papers.

Niels Kjølstad Poulsen was born in the central part of Zealand, Denmark in 1956.

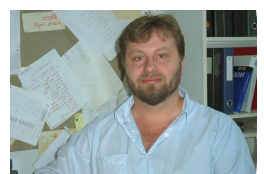

He received his M.Sc. and Ph.D. degrees in electrical engineering from The Institute of Mathematical Statistics and Operations Research (IMSOR), the Technical University of Denmark, in 1981 and 1984, respectively. He has been employed at the Technical university of Denmark from 1984, since 1990 as an associate professor at The Department of Applied Mathematics and Computer Science. His primary research interests is within stochastic control theory, system identification and fault diagnosis.

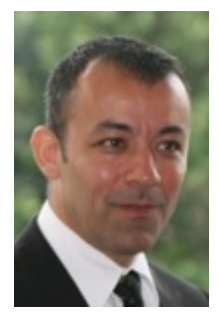

Illmar Ferreira Santos received the Dr.Ing. degree from the Technical University of Munich, in Germany, the dr. techn. degree from the Technical University of Denmark, and the livre-docente degree from State University of Campinas, Brazil. He is full professor at the Department of Mechanical Engineering of the Technical University of Denmark. He works in the field of design, monitoring, and control of electromechanical systems with emphasis on rotating machines. His research interests focus upon machinery dynamics, tribology, and control (mechatronics). 


\section{COPRIME REPRESENTATION}

The work presented in this paper exploits a coprime factorisation of the controller and plant in order to design residual signals for active fault detection. The fault free plant $\mathrm{G}(0)$ can be written as a coprime factorisation as shown in equation (36) and the controller $K$ can be represented as a coprime factorisation as shown in equation (37).

$$
\begin{array}{ll}
G(0)=N M^{-1}=\tilde{M}^{-1} \tilde{N}, & N, M, \tilde{M}, \tilde{N} \in \mathcal{R} \mathcal{H}_{\infty} \\
K(0)=U V^{-1}=\tilde{V}^{-1} \tilde{U}, & U, V, \tilde{V}, \tilde{U} \in \mathcal{R} \mathcal{H}_{\infty}
\end{array}
$$

Here the matrices denoted with a 0 are known as the left coprime factorisation while those without are the right coprime factorisation. For such a coprime factorisation as shown in equation (36) and equation (37) the bezout identity as shown in equation (38) is satisfied.

$$
\begin{aligned}
{\left[\begin{array}{ll}
I & 0 \\
0 & I
\end{array}\right] } & =\left[\begin{array}{cc}
M & U \\
N & V
\end{array}\right]\left[\begin{array}{cc}
\tilde{V} & -\tilde{U} \\
-\tilde{N} & \tilde{M}
\end{array}\right] \\
& =\left[\begin{array}{cc}
\tilde{V} & -\tilde{U} \\
-\tilde{N} & \tilde{M}
\end{array}\right]\left[\begin{array}{cc}
M & U \\
N & V
\end{array}\right]
\end{aligned}
$$

Using the coprime factorisation of the nominal plant $G(0)$ and controller $K$ it is possible to define all controller that stabilise the nominal plant as shown in equation (39).

$$
K(Q)=(\tilde{V}+Q \tilde{N})^{-1}(\tilde{U}+Q \tilde{M}), \quad Q \in \mathcal{R} \mathcal{H}_{\infty}
$$

It is possible to define an equivalent representation of all controller stabilising the nominal plant using the right factorisation as well. Such a controller can be implemented as shown on Figure 23.

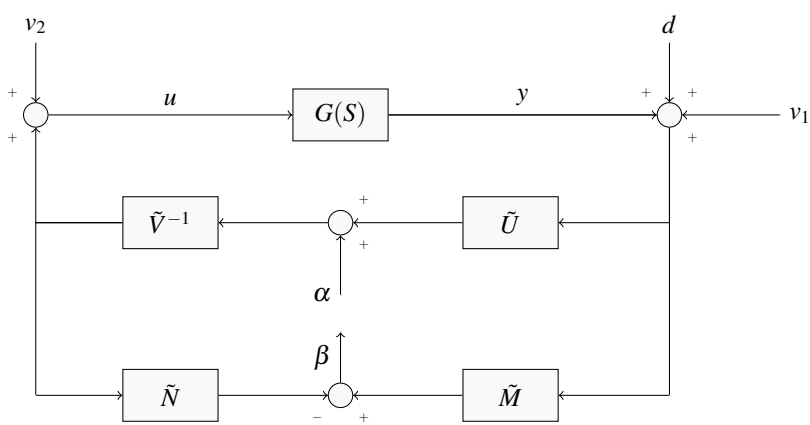

Fig. 23. Experimental test setup. $G(S)$ represents the gas bearing, $\alpha$ is the excitation signal and $G$ is the nominal model of the gas bearing.
Here $S$ describe the system dynamics diverging from the nominal model, which in this paper is known as the system faults. How the system $S$ influence the plant is shown in equation (40) using the right coprime factorisation and in equation (41) using the left coprime factorisation.

$$
\begin{array}{ll}
G(S)=(N+V S)(M+U S)^{-1}, & S \in \mathcal{R} \mathcal{H}_{\infty} \\
G(S)=(\tilde{N}+S \tilde{V})^{-1}(\tilde{M}+S \tilde{U}), & S \in \mathcal{R} \mathcal{H}_{\infty}
\end{array}
$$

Furthermore the two signals $\alpha$ and $\beta$ has been introduced on Figure 23 which are used for injecting the excitation signal used for active fault detection and design of the residual generator. It was shown in [24] that for a system as shown in Figure 23 the transfer functions from the inputs to outputs are given as shown in equation (42).

$$
\left[\begin{array}{l}
y \\
u \\
\beta
\end{array}\right]=T_{c l}(S)\left[\begin{array}{l}
d \\
v_{1} \\
v_{2} \\
\alpha
\end{array}\right]
$$

Where $T_{c l}$ is

$$
\left[\begin{array}{cccc}
(N+V S) \tilde{U} & (N+V S) \tilde{U} & (N+V S) \tilde{V} & N+V S \\
(M+U S) \tilde{U} & (M+U S) \tilde{U} & (M+U S) \tilde{V} & M+U S \\
\tilde{M}+S \tilde{V} & \tilde{M}+S \tilde{V} & \tilde{N}+S \tilde{U} & S
\end{array}\right]
$$

With the controller designed it is possible to calculate the Youla-Kucera factorisation. One such factorisation for a P-controller can be found in [27] and is given in equation (43) for the right coprime factorisation and in equation (44) for the left coprime factorisation.

$$
\begin{aligned}
{\left[\begin{array}{cc}
M & U \\
N & V
\end{array}\right] } & =\left(\begin{array}{c|cc}
A+B F & B & 0 \\
\hline F & I & K \\
C & 0 & I
\end{array}\right) \\
{\left[\begin{array}{cc}
\tilde{V} & -\tilde{U} \\
-\tilde{N} & -\tilde{M}
\end{array}\right] } & =\left(\begin{array}{c|cc}
A+B P C & -B & B K \\
\hline F-P C & I & -K \\
C & 0 & I
\end{array}\right)
\end{aligned}
$$

Here $F$ is a fictitious gain that satisfy all eigenvalues of $A+B F$ are in the stable left half plane. Equivalently it is possible to calculate the Youla-Kucera factorisation for the observer based controller. For such a controller the right factorisation is shown in equation (45) and the left factorisation is shown in equation (46).

$$
\begin{gathered}
{\left[\begin{array}{cc}
M & U \\
N & V
\end{array}\right]=\left(\begin{array}{c|cc}
A+B F & B & -L \\
\hline F & I & 0 \\
C & 0 & I
\end{array}\right)} \\
{\left[\begin{array}{cc}
\tilde{V} & -\tilde{U} \\
-\tilde{N} & -\tilde{M}
\end{array}\right]=\left(\begin{array}{c|cc}
A+L C & -B & L \\
\hline F & I & 0 \\
C & 0 & I
\end{array}\right)}
\end{gathered}
$$


Here $F$ is the state feedback matrix and $L$ is the observer gain matrix. Using the coprime factorisation of controller and plant the augmented controller can be expressed as in equation (47).

$$
J_{k}=\left[\begin{array}{cc}
U V^{-1} & \tilde{V}^{-1} \\
V^{-1} & -V^{-1} N
\end{array}\right]
$$

For an augmented controller as presented in equation (47) it might seem that the order is higher than for the nominal controller. The order of the two controllers has been shown to be the same in [28]. In the case of the observer based control design the controller $J_{k}$ can be written in state space form as in equation (47).

$$
J_{k}=\left[\begin{array}{c|cc}
A+B F+L C+L D F & -L & B+L D \\
\hline F & 0 & I \\
-(C+D F) & I & -D
\end{array}\right]
$$

Since a full order observer has been implemented the order of the nominal controller is the same as for the plant. From equation (48) it is easy to see that the augmented controller has the same order as well why the computational effort is the same with the nominal controller and the augmented. 